\title{
LA INTERACCIÓN DE LOS HOTELES CON LAS REDES SOCIALES: UN ANÁLISIS DE LOS HOTELES DE CUATRO ESTRELLAS DE LA COMUNIDAD DE MADRID (ESPAÑA)
}

\author{
Gemma Cascales García* \\ Laura Fuentes Moraleda** \\ Javier De Esteban Curiel ${ }^{* * *}$ \\ Universidad Rey Juan Carlos. Madrid
}

\section{RESUMEN}

En el presente trabajo se analiza como los hoteles de cuatro estrellas de la Comunidad de Madrid interaccionan con las redes sociales. En concreto, se comprueba que, a pesar de que el uso del social media está generalizado entre los hoteles de la región, la gestión del mismo para obtener beneficios por medio de las ventas o para manejar la reputación on line no está tan generalizada. Se muestra como la mayor parte de los hoteles participantes en el estudio aprovechan las redes sociales como canal de comunicación pero que los hoteles pertenecientes a cadenas utilizan más las redes como canal de ventas.

Palabras clave: hoteles de cuatro estrellas, redes sociales, social media, Facebook, Tripdavisor, twitter.

Recibido: 12 de mayo de 2015

Devuelto para su revisión: 5 de febrero de 2016

Aceptado: 30 de abril de 2016

* Programa Ciencias Sociales y Jurídicas. Escuela Internacional de Doctorado. E-mail: g.cascalesg@alumnos.urjc.es

** Departamento de Economía de la Empresa. Universidad Rey Juan Carlos, Madrid (España). Campus de Fuenlabrada. Camino del Molino s/n. 28943 Fuenlabrada. MADRID (España). E-mail: laura.fuentes@urjc.es

*** Departamento de Economía de la Empresa. Universidad Rey Juan Carlos, Madrid (España). Campus de Vicálvaro. Paseo de los Artilleros, s/n. 28032 MADRID (España).E-mail: javier.deesteban@urjc.es 


\title{
Interaction of hotels with social networks: an analysis of the four star hotels in Madrid Region, Spain
}

\begin{abstract}
This paper will analyze how the four star hotels in Madrid interact with social networks. In particular, it is found that, despite the use of social media is widespread among hotels in the Madrid region, its management for profit through sales or to manage online reputation is not as widespread. It is shown as most of the participating hotels take advantage of social networks as a communication channel but the hotels belonging to chains used widely as social media as a sales channel to generate direct revenues in the hotel.
\end{abstract}

Keywords: four star hotels, social networks, Facebook, Tripadvisor, Twitter, social media.

\section{INTRODUCCIÓN}

La rápida evolución de las Tecnologías de la Información y Comunicación (a partir de ahora TICs $)^{1}$ (Becerril, 2007) es, hoy en día, un hecho incuestionable; se tardaron más de 35 años en conseguir 50 millones de usuarios en la Radio, en la Televisión 13 años, Internet 4 años, y Facebook tan sólo tardó 9 meses en conseguir 100 millones de usuarios.

Las TICs han revolucionado todas las disciplinas y áreas de conocimiento, especialmente aquellas relacionadas con la comunicación y hoy día son consideradas como un fenómeno mundial que muchos tratan de explicar (Kotler, 2004). Los grandes operadores y canales de comunicación, están viendo cómo están perdiendo audiencia, y en cambio, portales como Facebook, Youtube, Twitter, están registrando millones de nuevos registrados cada año y millones de contenidos vistos (Arenas, 2010; Barranco, 2000).

Sectores como el del transporte, textil, alojamiento, comunicaciones, entre otros, han sufrido grandes transformaciones en el modo en el que se comunican y ofrecen sus productos o servicios a sus clientes. En el caso de la actividad turística, la transformación ha sido especialmente significativa, ya que tanto destinos como empresas y turistas, en pocos años han presenciado como han cambiado sus patrones de comportamiento en el proceso de compra y consumo de los servicios turísticos. En el sector turístico, las tecnologías en la distribución han producido un cambio de rumbo en el antiguo sistema (Fuentes, Figueroa y Balthasar, 2003), lo que es especialmente relevante para países como España donde el sector turístico, es el responsable del 10,9\% del Producto Interior Bruto y del $12 \%$ del empleo (Ministerio de Industria, Comercio y Turismo, 2015).

El presente artículo se centra en el sector turístico, concretamente en el del alojamiento y más específicamente en los hoteles de cuatro estrellas ubicados en Madrid. Las razones que justifican la elección de este tipo de hoteles en esta región, son básicamente tres. La primera se basa en la importancia de la actividad turística de la Comunidad de Madrid: la

1 Las Tecnologías de la Información y la Comunicación son el conjunto de tecnologías desarrolladas para gestionar información y enviarla de un lugar a otro (Becerril, 2007). 
región madrileña representa uno de los focos turísticos más importantes del país con cifras superiores a los 8.000 millones de euros en volumen de negocios de hostelería y cuenta, según los datos del Instituto Nacional de Estadística en 2012, con 413 establecimientos de hoteleros (estrellas oro) abiertos (tomando el máximo dato mensual de establecimientos abiertos en 2012). En segundo lugar, porque los hoteles de cuatro estrellas son los más numerosos en la región: del total de establecimientos, 178 son establecimientos de cuatro estrellas, representando la mayor proporción entre los establecimientos de alojamiento de la región (43\% respecto al total de hoteles) por delante de los hoteles de tres estrellas que en 2012 representaban en la región el 31,7\% de los establecimientos hoteleros abiertos y también por delante de los de cinco estrellas que representan el 6,2\%. En tercer lugar, los indicadores de rentabilidad de los hoteles de cuatro estrellas en la Región de Madrid publicados por el Instituto Nacional de Estadística, reflejaban en 2012 una ocupación por habitación del 62,36\%, una tarifa media diaria de 78,3€ y un RevPar (ingreso por habitación disponible) de 45,4€. Estos datos reflejaban la situación complicada por la que han pasado los hoteles de la Comunidad Madrid y es un hecho que apoya la necesidad de estudiar cómo mejorar la rentabilidad de los establecimientos hoteleros en momentos de crisis.

Bajo estas circunstancias, el objetivo del trabajo es conocer cómo los hoteles de categoría cuatro estrellas de la Comunidad de Madrid se están adaptando a los cambios que las redes sociales han provocado en el modo de comunicarse con los consumidores. Para ello, la investigación se articula alrededor de cuatro partes. Tras la presente introducción se desarrolla la revisión de la literatura sobre el marketing y las redes hoteles y el turismo, concretamente en el sector hotelero. Esta revisión, que además permite la formulación de la hipótesis del trabajo, pone de manifiesto por un lado el interés de las redes sociales en diferentes sectores de actividad turística y también cómo estos canales online han modificado la forma de interactuar con los clientes, de comunicarse con ellos y de gestionar su reputación. La segunda parte contiene la explicación de la metodología llevada a cabo, que se basa fundamentalmente en un estudio cuantitativo para ahondar en el conocimiento, participación y usos específicos en redes sociales de los hoteles de cuatro estrellas de Madrid. En la tercera parte se exponen los resultados principales y en el último bloque, se desarrollan las conclusiones del trabajo y las implicaciones prácticas de las mismas para el sector de los hoteles de cuatro estrellas de la Comunidad de Madrid.

\section{LAS REDES SOCIALES}

Las plataformas sociales como Facebook o Twitter han cambiado las condiciones de la participación de los usuarios en el desarrollo de los diferentes servicios. Los usuarios activos de las comunidades, el rápido desarrollo interactivo de las mismas, la facilidad con la que los usuarios desarrollan nuevas aplicaciones, y el bajo coste de distribución son algunas de las conocidas características que han proporcionado las nuevas tecnologías sociales (Johnson y Hyysalo, 2012).

Hagen y Robertson (2010) analizan cuáles son los cambios y las oportunidades de participación de los usuarios en el contex to social de las nuevas tecnologías, y las define como herramientas y prácticas que constituyen un incremento de las capacidades del personal 
como la comunicación, producción, publicación, distribución y difusión. Ejemplo de estas tecnologías sociales son las plataformas de Facebook, Ning, Flickr, Youtube, Wordpress.

Los puntos en común que tienen todas estas plataformas sociales son que el uso del contexto es variable y complejo, que sus diseños son nuevos y atractivos, los roles asignados, y la fácil naturaleza del diseño y la utilización. El anonimato de los usuarios y la distribución geográfica también son nuevos cambios a tener en cuenta (Clement, Constantino, Kurtz y Tissenbaum, 2008; Ehn, 2008).

En contraste con los tradicionales modelos de ingeniería de software, en estas plataformas, la fase de mantenimiento se completa con el diseño que aportan los usuarios (Henderson y Kyng, 1992; Voss, 2009). Esta posibilidad que permite diseñar a los usuarios, proporciona a las redes sociales las características de apropiación, personalización y adaptación (Balka y Wagner, 2006; Morch, 1997; Nardi, 1993; Fundación Banesto, 2011).

Por tanto, las ventajas de las redes sociales radican en tres aspectos fundamentales; (i) el fácil acceso a las actividades online, (ii) los foros online, (iii) y las estadísticas en tiempo real (Johnson y Hyysalo, 2012; Anderson, 2012; Hsu, 2012). Se trata de plataformas muy fáciles de manejar, casi intuitivas, donde el rango de las opciones que ofrecen dependen de cada red social, pero algunas son comunes como las de encontrar personas/ amigos/contactos, enviar mensajes privados, observar los perfiles de los usuarios, entre otras (Hsu, 2012). Algunas de las más conocidas son Facebook, Twitter y TripAdvisor (usada habitualmente en el sector turístico):

(i) Facebook es una plataforma social creado por Mark Zuckerberg² y fundado por Eduardo Saverin, Chris Hughes, Dustin Moskovitz y Mark Zuckerberg. Originalmente era un sitio para estudiantes de la Universidad Harvard, pero actualmente está abierto a cualquier persona que tenga una cuenta de correo electrónico (Jaramillo, 2011). Facebook es una herramienta de marketing viral (Holzner, 2009; Del Pino, 2007; Sivera, 2008) y se ha convertido en la primera red social por número de usuarios y volumen de conexiones (Hsu, 2012), debido a la diversidad de aplicaciones que Facebook ofrece y la innovación constante que se aplica a sus aplicaciones (Treadaway y Smith, 2010; Benet, 2013). Ofrece una gran gama de usuarios desde los desarrolladores, los publicitarios, las empresas y los que lo utilizan en su vida personal. (Hsu, 2012; Benet, 2013). Según el estudio de Villalba, Martínez y Martínez (2014) sobre la promoción turística Internet, la red social más utilizada por los portales turísticos es Facebook. Los hoteles pueden tomar las ventajas de Facebook ya que es una red social no permanente (Treadaway y Smith, 2010) y rápida (Hsu, 2012).

(ii) Twitter es un sitio web de microblogging que permite a sus usuarios enviar y leer "microentradas" de texto de una longitud máxima de 140 caracteres denominados tweets (Jaramillo, 2011). Estas actualizaciones se muestran en la página de perfil

2 Mark Elliot Zuckerberg (14 de mayo de 1984, White Plains, Estados Unidos), más conocido como Mark Zuckerberg, es un programador y empresario estadounidense conocido por ser el creador de Facebook, Para desarrollar la red, Zuckerberg contó con el apoyo de sus compañeros de Harvard, el coordinador de Ciencias de la computación y sus compañeros de habitación Eduardo Saverin, Dustin Moskovitz y Chris Hughes. (Facebook, 2010) 
del usuario, y también se envían de forma inmediata a otros usuarios o solo a su círculo de amigos (Rosero, 2013). La gran revolución de Twitter fue el tiempo real de sus tweets, creando así una adicción en los seguidores que esperan impacientes esas comentarios (Jaramillo, 2011) ya que los hashtags se han convertido en el amigo inseparable de los tuiteros (González, 2013 ${ }^{\mathrm{a}}$ ). Si se une la rapidez y la viralidad con la que se transmite la información, al uso de los teléfonos de nueva generación, lo hace una herramienta muy potente (Ferreras, 2011). Twitter actualmente está considerada como una de las páginas Web 2.0 más populares, con más de 100 millones de usuarios (Twitter, 2014; Muñoz y Argüelles, 2010).

(iii) Tripadvisor es una de las más exitosas redes sociales de turistas donde las recomendaciones de los viajeros y las internacionales críticas superan los 24 millones de usuarios al mes (GlobRes, 2009). El potencial daño que puede realizar las críticas negativas de los usuarios se contrarresta si el hotel contesta a los usuarios e intenta mejorar y superar las expectativas del viajero (Buhalis y Law, 2008) reforzando la marca (GlobRes, 2009; Cox, 2009; Bulchand - Gidumal et al., 2013).

\section{EL EMPLEO DE LAS REDES SOCIALES EN EL SECTOR HOTELERO}

Las redes sociales han revolucionado el modo en que los turistas consumen productos y servicios turísticos, la forma de hacer reservas, la de informarse sobre las nuevas ofertas, o sobre los posibles planes e itinerarios así como la forma de comunicarse con el proveedor de servicios (Phelan, Chen y Haney, 2013).

Además, las redes sociales facilitan a los hoteles conocer más sobre sus clientes y adaptar sus mensajes a las nuevas demandas de consumidores (Hsu, 2012; Ayeh, Leung, Au y Law, 2012), ayudando a crear relaciones estrechas en un mundo virtual (Zarella, 2010). Los hoteles pueden crear sus propios perfiles sociales o trabajar desde otros portales de viajes (Hsu, 2012). Según autores como Braun y Hollick, 2006 y Aces, 2010, los hoteles deben desarrollar sus habilidades relacionadas con el marketing en las redes sociales, para crear experiencias en los usuarios que beneficio a su entorno virtual social.

Los hoteles pueden obtener beneficio usando las plataformas sociales. Las redes sociales como Facebook, pueden aportar un gran número de usuarios y los hoteles pueden crear estrategias internacionales a través de los consumidores que son llamados "fans", que será exitosa si los hoteles realizan un presencia activa en la red, generando contenido, contestando las preguntas de los usuarios y respondiendo a los comentarios (Hsu, 2012). Sin embargo, en términos generales, los hoteles no están optimizando todos los recursos que Facebook les brinda, ya que este no es un canal solo para publicar promociones, sino que las interacciones con el cliente deben ir más allá (Chan y Guillet, 2011; Hosteltur, 2013). Algunos hoteles no le están dando importancia a las preguntas que los usuarios de Facebook les hacen a través de esta plataforma (Chan y Guillet, 2011). Además, no responder a estos comentarios, puede suponer una percepción negativa sobre la compañía (eMarketer, 2012b).

Google también ayuda a los hoteles a fomentar las reservas de los usuarios a través del producto Hotel Finder de Google Travel (Sena, McNellis y McDade 2014). Google Finder ofrece una experiencia de búsqueda para los usuarios de los proveedores de reservas que hasta 
ese momento pagaban grandes cantidades de dinero por el posicionamiento y anuncios en google (Sena et al, 2014). Según un estudio de Google Travel y Hotel Finder, hay que dotar de mayor experiencia en la reserva, ya que según Google, los viajeros emplean 55 minutos para reservar un hotel y un vuelo, visitan una media de 17 páginas y hacen clic en cuatro anuncios por cada búsqueda, y para esto el $90 \%$ de los internautas utilizan múltiples pantallas.

Existen estudios recientes que identifican las relaciones entre el turista y la empresa hotelera a través de plataformas sociales y como se mejoran aspectos relacionados con la fidelidad, la confianza y la satisfacción del cliente. (Kasavana, Nusair, y Teodosic, 2010; Verhoef, Reinartz y Krafft, 2010; Xing y Gratel, 2010). Otros trabajos analizan el impacto de Internet en la actividad turística (Villalba et al , 2014 cit: Castells y Díaz, 2001; Pérez, Martínez y Abella, 2002; Heung, 2003; Stamboulis y Skayannis, 2003).

Sin embargo, no existen tantos trabajos que analicen el uso de las redes sociales por parte del sector hotelero, y mucho menos, estudios que comparen el uso que hacen de las redes sociales los hoteles independientes y los pertenecientes a cadenas. Uno de los pocos trabajos que ha analizado el empleo de las redes sociales en el sector hotelero español ha sido estudiado por Mota (2012), según el cual los hoteles emplean redes sociales en un $73 \%$. Sin embargo, a pesar de que la inmersión en redes sociales por parte de los hoteles puede ser amplia, probablemente debido su facilidad de uso y su bajo coste, el uso eficiente de las mismas puede no serlo, principalmente en los hoteles independientes (Mellinas, Martínez y Bernal, 2014).

El estudio de Mellinas, Martínez y Bernal (2014) sobre el uso de las redes sociales en los hoteles de la costa española concluye que las grandes cadenas hoteleras han asumido correctamente el uso y la gestión de las redes sociales en su inmensa mayoría, pero aproximadamente un tercio del resto de hoteles no acaban de incorporarse a las redes sociales. Además, los autores observan que la presencia de hoteles independientes en redes sociales presenta graves deficiencias en la mayoría de los casos estudiados. Para los establecimientos pertenecientes a pequeñas cadenas, las cifras también indican una gestión inadecuada en un alto porcentaje. Mellinas et al (2014), concluyen que los hoteles deberían concienciarse de que la mayoría de sus clientes utiliza Internet durante el proceso de compra y que la mayoría de esos clientes son también usuarios de redes sociales.

Por tanto, los responsables comerciales de los establecimientos de alojamiento deberían empezar a hacer un uso avanzado de las redes sociales por los numerosos beneficios que pueden reportar a los hoteles, relacionados con la (i) comunicación con sus clientes actuales y potenciales; (ii) con la realización de ventas y (iii) con su reputación en la red:

(i) Comunicación y las redes sociales. Las comunicaciones a través de los portales sociales permiten que la relación entre los clientes y los hoteles sea directa y cercana a pesar de la distancia geográfica (Hsu, 2012) ya que las redes sociales ayudan a los hoteles a transmitir su mensaje en la red (O'Connor, Hopken y Gretzel, 2008; Albee, 2010; Ayeh et al., 2012). Estos portales de tecnología social están creando grandes comunidades caracterizadas por su alto grado de participación (algunas ya triplican la población española) lo que está planteando nuevas formas de hacer marketing y promoción, y muy especialmente en la manera de relacionarse con los clientes (Sule y Prieto, 2010). 
Las webs 2.0 y las plataformas sociales son las herramientas más apropiadas para las comunicaciones con los clientes (Holzner, 2009; Chan y Guillet, 2011). Los expertos (Hsu, 2012) recomiendan a los hoteles la ventaja de usar plataformas como Facebook ya que pueden obtener un mayor conocimiento de sus clientes para poder enviarles después información comercial más depurada y efectiva. Estudios realizados indican que las websites de los hoteles aún no incorporan componentes sociales que puedan ayudar a interactuar con los clientes (Gilbert, Powell-Perry y Widijoso, 1999; Arnott and Bridgewater, 2002: Bai, Hu y Jang, 2007; Li y Wang, 2010; Dwivide, Yadav y Ventkatesh, 2012).

(ii) Ventas a través de las redes sociales. Las estrategias de marketing a través de redes sociales son muy importantes para los hoteles ya que pueden asegurar las ventas gracias a la conexión e interacción con los consumidores de todo el mundo (Braun, 2002). En este sentido, un estudio de la Comisión Europea del Turismo (2010) estableció la necesidad de crear políticas de eMarketing para los hoteles.

Los beneficios de utilizar estas técnicas del marketing son entre otras, la facilidad para poder cambiar precios, incluso de forma diaria, lo que se denomina yield management pricing, y que es utilizado de forma habitual por las empresas de alojamiento y las compañías aéreas. Además los usuarios serán más conscientes de los precios, ya que en muchos casos será un factor determinante, (Kotler et a, 2006:456) por lo que la transparencia en la prestación de servicios, y en la diferenciación del valor añadido jugará un papel fundamental. Otro de los beneficios para las empresas serán la reducción de costes intermediarios, la construcción de relaciones con los clientes y/o usuarios, y la medición del tamaño de su audiencia. Para los consumidores los beneficios también son palpables. Pueden ejercer su derecho a comprar las 24 horas del día, encontrando información de forma más rápida y precisa (Kotler y Kartajaya, 2010:457). Recientes estudios han demostrado que las buenas calificaciones en las redes sociales tienen impacto directo en las ventas (Ye et al, 2011).

(iii) Reputación online. El concepto reputación online se define como lo que se dice sobre algo o alguien y cómo esto afecta a la forma en la que los grupos de interés (stake holders) interactúan con él (Cuesta y Gaspar, 2013). En el ámbito turístico, la reputación online ha obligado a los directivos hoteleros una readaptación a esta nueva realidad (Martínez, Bernal y Pedro, 2012).

Los turistas, cuando van a tomar la decisión de compra sobre un alojamiento, tienen hoy a su disposición un sinfín de portales online donde obtener información sobre los establecimientos, y sobre todo encuentran comentarios de otros usuarios que pueden alterar su decisión final de compra, es decir, se basan en la reputación online del hotel para decidir (Diana, 2014). Por ello, es fundamental la disposición y capacidad de los hoteles para responder de forma eficiente y efectiva a las críticas efectuadas por los usuarios (Callarisa, Sánchez-García, Cardiff y Roshchina, 2012). Según McGuirre y Noone (2014), existen determinados atributos que tienen un rápido y significativo impacto en las opiniones de los usuarios, y cambian dependiendo de si el viajero es vacacional o de negocios; En el caso de los turistas de negocios, los aspectos más relevantes son (i) comentarios; (ii) valor de la marca; (iii) 
comparación entre la competencia; (iv) precio y (v) críticas emocionales. En el caso de los turistas vacacionales, los aspectos más relevantes son (i) comentarios; (ii) precio; (iii) comparación entre la competencia; (iv) ranking de Tripadvisor y por último (v) el valor de la marca. Para ambos tipos de turistas, los comentarios son el atributo que más influye en los usuarios, por ser el que más confianza les proporciona.

Una vez que se ha evidenciado la importancia que tienen las nuevas plataformas digitales para la actividad turística y una vez que se han puesto de relieve las distintas utilidades que pueden brindar estas redes sociales al sector hotelero como nuevos canales de comunicación, venta y como modo de gestionar la reputación on line, este trabajo se centra en conocer cuál es la situación de los hoteles de la Comunidad de Madrid y más específicamente los de cuatro estrellas y si existen diferentes situaciones entre los hoteles que pertenecen a una cadena y los que son independientes. Concretamente, los objetivos perseguidos son:

(i) Determinar la importancia que tanto los hoteles de gestión independiente como los de cadena otorgan a las redes sociales, así como analizar si la pertenencia o no a una cadena está relacionado con la importancia otorgada a las redes sociales o social media. El primer objetivo se concreta en la contrastación de la siguiente hipótesis H1. La pertenencia de los hoteles de cuatro estrellas de la Comunidad de Madrid a una cadena está relacionado con la asignación del nivel de importancia otorgada al uso de las redes sociales.

(ii) Explorar el uso que los gestores de los distintos hoteles realizan de las redes sociales. Concretamente, se pretende conocer si los hoteles de cuatro estrellas de la Comunidad de Madrid pertenecientes a una cadena hacen un mayor uso de las redes sociales como canal de comunicación y como canal de ventas que los hoteles independientes de la región de la misma categoría. También se pretende analizar si los hoteles de cuatro estrellas de la Comunidad de Madrid pertenecientes a una cadena miden sus actividades en las redes sociales más que los hoteles independientes de la región de la misma categoría ${ }^{3}$.

\section{METODOLOGÍA}

La metodología llevada a cabo en el presente trabajo se apoya en un estudio cuantitativo basado en una encuesta realizada a los hoteles de cuatro estrellas de la Comunidad de Madrid, con el objetivo de saber la importancia que le otorgan al social media y el grado de aplicación en su gestión.

La población objeto de este estudio se compone los hoteles de 4 estrellas de Madrid, y que pertenecían a la AEHM en el momento de la realización del trabajo (137 hoteles). De los hoteles de cuatro estrellas de la Comunidad de Madrid pertenecientes a la AEHM, tan solo 26,27\% eran independientes o no estaban unidos a ningún grupo o representante hotelero. De ellos, el 41,66\% están representados en el estudio.

3 El segundo objetivo por su propia naturaleza, de carácter exploratorio, no requiere de la formulación de hipótesis. 
Se administró un cuestionario con preguntas cerradas que fue remitido por correo electrónico a los distintos directores de todos los hoteles. Finalmente, respondieron al cuestionario un total de 105 hoteles. Para la realización de este trabajo se contó con el apoyo de la Asociación Empresarial de Hosteleros de Madrid (AEHM) y durante el periodo de recogida de la información, se comprobó personalmente que cada cuestionario fue respondido por el director o por la persona del hotel encargada del manejo de las redes sociales en el establecimiento.

El cuestionario estaba formado por 15 preguntas; de las que el $80 \%$ trataban aspectos sobre social media y el $20 \%$ sobre las características del establecimiento (pertenencia a cadena, ubicación, número de habitaciones). Para analizar los datos obtenidos se ha empleado el programa estadístico de SPSS.

Tabla 1

\section{FICHA TÉCNICA DE LA INVESTIGACIÓN}

\begin{tabular}{ll}
\hline \multicolumn{2}{c}{ Características de la población y la muestra } \\
\hline Población objeto de estudio & Hoteles de cuatro estrellas \\
Ámbito geográfico & Comunidad de Madrid, pertenencia a la AEHM ${ }^{4}$ \\
Recogida de la información & Encuesta. Administrada por correo electrónico \\
Tratamiento de la información & Programa estadístico SPSS \\
Población Finita & 137 hoteles de cuatro estrellas de la CAM \\
Tamaño de la muestra & 105 hoteles de cuatro estrellas de la CAM \\
Tasa de respuesta & $73,76 \%$ \\
Error muestral & $+/-4,72 \%$ \\
Nivel de confianza & $95 \%$ p=q=0,5) \\
Periodo de recogida de la información & Marzo-Mayo 2012 \\
Duración del cuestionario & $7-9$ minutos \\
Técnicas estadísticas utilizadas & Análisis descriptivo. Chi Cuadrado y Test de Fisher \\
\hline
\end{tabular}

Fuente: elaboración propia.

\section{PRINCIPALES RESULTADOS}

A continuación se muestran los principales resultados del estudio cuantitativo llevado a cabo en esta investigación. Después de la descripción de las características básicas de los hoteles participantes en el estudio, se exponen los resultados divididos en dos bloques: (i) conocimiento, importancia y uso de redes sociales por parte de los hoteles y (ii) empleo específico de redes sociales (comunicación con clientes, ventas, control de resultados)

4 Asociación Empresarial Hotelera de Madrid. 


\subsection{Características básicas de la muestra}

Como se ha comentado anteriormente, la muestra seleccionada para el trabajo fueron los hoteles de cuatro estrellas pertenecientes a la Asociación Empresarial de Hosteleros de Madrid (137 hoteles). De la totalidad de la muestra, se obtuvo respuesta de 105 hoteles (el 73,76\%). De la muestra analizada, el 14,3\% eran hoteles independientes, mientras que el $85,7 \%$ pertenecían a algún grupo o cadena. En cuanto a la capacidad, el 47,6\% tienen más de 100 habitaciones, mientras que el 41,9\% tienen entre 50 y 100 habitaciones, según se muestra en la tabla 2. La mayor parte de los hoteles encuestados estaban ubicados en el centro de Madrid (71,4\%).

Tabla 2

CARACTERIZACIÓN DE LA MUESTRA

\begin{tabular}{|c|c|c|c|}
\hline & $\begin{array}{c}\text { Hoteles } \\
\text { Independientes }\end{array}$ & $\begin{array}{l}\text { Hoteles de } \\
\text { Cadena }\end{array}$ & Total \\
\hline Hoteles de cuatro estrellas pertenecientes a la AEHM (2012) & 36 & 101 & 137 \\
\hline Encuestas recibidas & 15 & 90 & 105 \\
\hline $\begin{array}{l}\text { Tasa de respuesta (sobre los hoteles de cuatro estrellas de la } \\
\text { AEHM en 2012) }\end{array}$ & $41,66 \%$ & $89 \%$ & $76,64 \%$ \\
\hline $\begin{array}{l}\text { Tamaño hoteles ( } \mathrm{n}^{\circ} \text { habitaciones): } \\
0-50 \text { habitaciones } \\
50-100 \text { habitaciones } \\
\text { Más de } 100 \text { habitaciones }\end{array}$ & $\begin{array}{l}3 \\
5 \\
7\end{array}$ & $\begin{array}{c}8 \\
39 \\
43\end{array}$ & $\begin{array}{l}11 \\
44 \\
50\end{array}$ \\
\hline $\begin{array}{l}\text { Ubicación de los hoteles: } \\
\text { Periferia } \\
\text { Centro }\end{array}$ & $\begin{array}{l}27 \\
63\end{array}$ & $\begin{array}{c}3 \\
12\end{array}$ & $\begin{array}{l}30 \\
75\end{array}$ \\
\hline
\end{tabular}

Fuente: elaboración propia.

\section{(i) Conocimiento, importancia y uso de redes sociales}

De los encuestados, el $100 \%$ conocía lo que es y representa un community manager. Por otro lado, casi la totalidad $(98 \%)$ aprovechaba Internet y las redes sociales como canal de comunicación. Además, la mayor parte confirmó la existencia de una web 2.0 en su hotel, es decir, interactuaban directamente con el cliente a través de la web del hotel. Tan solo un $15 \%$ no contestó nada.

En cuanto a la importancia otorgada al manejo de las redes sociales, a los encuestados se les pidió que otorgasen dos niveles de importancia (siendo 1 algo importante y 2 muy importante) a las siguientes redes sociales: Tripadvisor, Web propia, Facebook, Twitter, Trivago, Otros. La mayor parte de los hoteles otorgaron más importancia a la web del hotel y a Tripadvisor.

Para determinar la existencia de posibles relaciones entre la importancia otorgada a las redes sociales y a la web, y la pertenencia o no del establecimiento a una cadena hotelera, 
se ha realizado un contraste de independencia (Test de Fisher ${ }^{5}$ ). En la tabla 3 se presentan en negrita aquellas redes sociales en las que se rechaza la hipótesis nula por tener un nivel de significación inferior o igual a $\mathbf{0 , 0 5}$. En dicha tabla se observa que existe relación entre las valoraciones de las diferentes redes sociales por parte de los hoteles en función de si pertenecen a una cadena o si son hoteles independientes, salvo en el caso de Facebook y Twitter donde no hay relación entre la importancia otorgada a las redes sociales y si el hotel pertenece a una cadena o es independiente.

Tabla 3

IMPORTANCIA OTORGADA AL MANEJO DE REDES SOCIALES Y WEB DEL ESTABLECIMIENTO

\begin{tabular}{|c|c|c|c|c|}
\hline \multirow{2}{*}{$\begin{array}{c}\text { Red social y } \\
\text { web }\end{array}$} & \multirow[t]{2}{*}{ Tipo de hotel } & \multicolumn{2}{|c|}{ Importancia } & \multirow{2}{*}{$\begin{array}{l}\text { Sig. asintótica } \\
\text { (bilateral) }\end{array}$} \\
\hline & & Algo importante & Muy importante & \\
\hline \multirow{2}{*}{ TripAdvisor } & Hotel independiente & 10 & 5 & \multirow{2}{*}{0,003} \\
\hline & Hotel de Cadena & 86 & 4 & \\
\hline \multirow{2}{*}{ Web } & Hotel independiente & 11 & 4 & \multirow{2}{*}{0,008} \\
\hline & Hotel de Cadena & 87 & 3 & \\
\hline \multirow{2}{*}{ Facebook } & Hotel independiente & 3 & 12 & \multirow{2}{*}{0,453} \\
\hline & Hotel de Cadena & 14 & 76 & \\
\hline \multirow{2}{*}{ Twitter } & Hotel independiente & 8 & 7 & \multirow{2}{*}{0,133} \\
\hline & Hotel de Cadena & 31 & 59 & \\
\hline \multirow{2}{*}{ Trivago } & Hotel independiente & 12 & 3 & \multirow{2}{*}{0,010} \\
\hline & Hotel de Cadena & 40 & 50 & \\
\hline
\end{tabular}

Fuente: elaboración propia.

Los resultados anteriores permiten aceptar la hipótesis planteada, ya que según los datos, existe relación entre la importancia otorgada a dos de las redes sociales y a la web y la pertenencia del hotel a una cadena.

En cuanto al canal que han habilitado para ponerse en contacto con sus clientes, "Pregunta 14. ¿Cómo se comunica con los clientes?” las cadenas hoteleras han seleccionado la opción de su propia web, Facebook y Tripadvisor con un porcentaje en torno al 96\%.

Por otro lado, según se puede apreciar en la siguiente tabla 4, existen diferencias en el canal empleado para comunicarse con los clientes por parte de los hoteles de cadena respecto de los independientes tanto en el uso de la web como en el uso de Facebook y de TripAdvisor. Esto puede deberse, en gran medida a la poca confianza que le otorgan a la propia web y al resto de canales los hoteles independientes.

5 El test exacto de Fisher es una prueba de significación estadística utilizada en el análisis de tablas de contingencia. 
Tabla 4

CANAL PARA COMUNICARSE CON LOS CLIENTES

\begin{tabular}{|c|c|c|c|}
\hline & & $\begin{array}{c}\text { Hoteles de } \\
\text { Cadena }\end{array}$ & $\begin{array}{c}\text { Hoteles } \\
\text { Independientes }\end{array}$ \\
\hline Canal de comunicación con los clientes & & Frecuencias & Frecuencias \\
\hline \multirow{2}{*}{ Web del hotel } & SI & 86 & 9 \\
\hline & NO & 4 & 6 \\
\hline \multirow{2}{*}{ Blog } & SI & 50 & 6 \\
\hline & NO & 40 & 9 \\
\hline \multirow{2}{*}{ Facebook } & SI & 87 & 10 \\
\hline & NO & 3 & 5 \\
\hline \multirow{2}{*}{ Tripadvisor } & SI & 87 & 11 \\
\hline & NO & 3 & 4 \\
\hline
\end{tabular}

Fuente: elaboración propia.

Además, es importante mencionar que un gran porcentaje de los encuestados han contestado que Tripadvisor es el canal habitual a través del cual gestionan las críticas ("Pregunta 12. ¿Tiene establecido algún canal online para responder a las críticas de los clientes?"). Del mismo modo, el 45,71\% de los hoteleros utilizan otras vías diferentes a la red, posiblemente encuestas de calidad, buzón de sugerencias, envío de mails de seguimiento, etc.

(ii) Empleo específico de redes sociales (comunicación con clientes, ventas, control de resultados)

El segundo de los objetivos del trabajo es analizar el uso específico de las redes sociales que realizan los hoteles para comunicarse con los clientes y para mejorar las ventas. Según los resultados, la mayor parte de los hoteles encuestados emplean redes sociales tanto para comunicarse con los clientes y como canal de ventas. En la tabla 5 aparecen representadas las respuestas a las preguntas efectuadas en la encuesta por tipología de hotel.

Tal y como se desprende de la tabla siguiente, se observa la existencia de diferencias entre las respuestas otorgadas por los hoteles de cadena respecto a los independientes, salvo en el uso del social media como canal de comunicación, donde ambos tipos de hotel usan las redes sociales para comunicarse con clientes. Los hoteles pertenecientes a cadenas emplean de un modo más generalizado las redes sociales como canal de ventas, generan ingresos directos por medio de las redes sociales y realizan estudios y miden los resultados de las acciones llevadas a cabo en el social media. En este aspecto, y tal y como concluyen también Mellinas, Martínez y Bernal (2014), se aprecia como los hoteles de cadena tienen un desarrollo superior en el ámbito del social media respecto a los hoteles independientes de la misma categoría. 
Tabla 5

USO DE LAS REDES SOCIALES POR PARTE DE HOTELES INDEPENDIENTES Y DE CADENA. FRECUENCIAS

\begin{tabular}{lcccc}
\hline \multirow{2}{*}{ Cuestiones } & Respuestas & $\begin{array}{c}\text { Hoteles } \\
\text { independientes }\end{array}$ & $\begin{array}{c}\text { Hoteles } \\
\text { de cadena }\end{array}$ & Total \\
\hline \multirow{2}{*}{$\begin{array}{l}\text { ¿Aprovecha el social media como canal de } \\
\text { comunicación? }\end{array}$} & NO & 1 & 1 & 2 \\
\cline { 2 - 5 } $\begin{array}{l}\text { ¿Emplea las redes sociales como canal de } \\
\text { ventas? }\end{array}$ & SI & $\mathbf{1 4}$ & $\mathbf{8 9}$ & $\mathbf{1 0 3}$ \\
\cline { 2 - 5 } ¿Las redes sociales pueden generar ingresos & NO & 5 & 8 & 13 \\
\cline { 2 - 5 } directos en el hotel? & NO & $\mathbf{1 0}$ & $\mathbf{8 2}$ & $\mathbf{9 2}$ \\
\hline ¿Realizan estudios o miden resultados de las & SI & $\mathbf{1 1}$ & $\mathbf{8 6}$ & $\mathbf{9 7}$ \\
\cline { 2 - 5 } acciones llevadas a cabo en el social media? & NO & $\mathbf{8}$ & 8 & 16 \\
\hline ¿El futuro del social media pasa por las & NO & $\mathbf{7}$ & $\mathbf{8 2}$ & $\mathbf{8 9}$ \\
\cline { 2 - 5 } aplicaciones móviles? & SI & $\mathbf{1 4}$ & $\mathbf{9 0}$ & $\mathbf{1 0 4}$ \\
\hline
\end{tabular}

Fuente: elaboración propia.

Además se les preguntaba si, en su opinión en futuro del social media estaba en las aplicaciones móviles. A pesar de que un $92 \%$ del total de los hoteles participantes contestaron que sí, existen diferencias entre ambos grupos; de los hoteles de cadena solo contestaron que no un $10 \%$ mientras que los independientes contestaron que no un $46 \%$.

A raíz de los resultados, se sigue comprobando que el uso de las redes sociales es diferente en muchos aspectos cuando se trata de hoteles de cadena. Entre todos ellos destacan, por ser más diferentes las opiniones respecto a su importancia, la realización de estudios y medición de resultados de las acciones llevadas a cabo con el social media.

\section{CONCLUSIONES E IMPLICACIONES PRÁCTICAS}

Para finalizar este trabajo es conveniente destacar sus hallazgos más significativos para concretar las principales conclusiones e implicaciones prácticas. La primera conclusión del trabajo está relacionada con un hecho que hoy día es indiscutible: la importancia que tienen las nuevas plataformas digitales para la actividad turística, y concretamente para el sector hotelero, como nuevos canales de comunicación, venta y como modo de gestionar la reputación on line. La mayor parte de los hoteles que han formado parte de este trabajo reconocen su importancia y también afirman que las usan, probablemente debido a que su uso es relativamente fácil y su coste es bajo. Sin embargo, a pesar de la importancia que tienen y que le otorga el sector, es cierto que la gestión de las mismas todavía no es eficiente.

En relación con esto último la segunda conclusión del trabajo refleja que los hoteles objeto de estudio no se han adaptado plenamente a las redes sociales y muchos las emplean 
sólo como canal de comunicación. Además, aunque ambos tipos de hoteles (independientes y de cadena) hacen uso de las redes sociales como canal de comunicación, existen diferencias en el uso de las redes sociales en otros aspectos. Según los resultados, los hoteles regentados por cadenas hacen un mayor uso de las redes sociales como canal de ventas y como generador de ingresos directos. Además los hoteles pertenecientes a una cadena realizan estudios y mediciones de resultados de las acciones llevadas a cabo en el social media, lo que indica que las redes sociales se encuentran totalmente incorporadas a la estrategia general de estas empresas hoteleras. Por el contrario, estos resultados muestran que los hoteles de gestión independiente siguen siendo incapaces de explotar plenamente las redes sociales para maximizar sus beneficios.

Las implicaciones prácticas del estudio parecen sugerir que los hoteles independientes de cuatro estrellas de la Región de Madrid, deberían invertir en convertir la web en 2.0, para poder integrar las nuevas plataformas sociales. Además, es necesario que personal cualificado monitorice las acciones en las plataformas sociales, controlando la comunicación con los clientes, respondiendo a las posibles críticas o comentarios para mejorar la reputación online, y apostar por las plataformas sociales como nuevo canal de ventas.

La características de la muestra empleada hacen que las conclusiones no puedan ser trasladadas a la totalidad del conjunto de hoteles de la Comunidad de Madrid, pero, a pesar de ello, se mantiene que, partiendo de la existencia de diferencias entre hoteles de cadena e independientes, las empresas hoteleras deben utilizar más eficazmente las redes sociales. Está demostrado que representan un nuevo canal de comunicación y venta y ayudan a mejorar la reputación de los establecimientos con un coste inferior a los canales tradicionales.

\section{REFERENCIAS BIBLIOGRÁFICAS}

ALBEE, A. (2010): eMarketing Strategies for the Complex Sale. Marketing Interactions, INC., New York.

ANDERSON, C. (2012): The impact of social media on Lodging Performance, Vol. 12, No. 15, Conerll University, School of Hotel Administration.

ARENAS, M. (2010): «Redes sociales, ¿un virus sin cura?: las ventajas y los problemas para sus usuario» Agencia de Protección de Datos de la Comunidad de Madrid. Datospersonales.org, Madrid, 15 de Junio de 2012. Disponible en http://www.madrid.org/ cs/Satellite?c=CM_Revista_FP\&cid=1142581421779\&esArticulo=true\&idRevistaEle gida $=1142576007987 \&$ language $=$ es $\&$ pag $=1 \&$ pagename $=$ RevistaDatosPersonales $\% 2$ FPage\%2Fhome_RDP\&siteName=RevistaDatosPersonales] ISBN 1988-1797 No 43.

ARNOTT, D. C. y BRIDGEWATER, S. (2002): Internet, interaction and implications for marketing. Marketing Intelligence \& Planning, $\mathrm{n}^{\mathrm{a}}$ 20, pp. 86-95.

AYEH, J.K.; LEUNG, D.; AU, N. Y R. LAW (2012): «Perceptions and strategies of hospitality and tourism practitioners on social media: An exploratory study » Academia, 28 de octubre del 2014. Disponible en http://www.academia.edu/1286988/Perceptions_and_strategies_of_hospitality_and_tour_ism_practitioners_on_social_media.

BAI, B; HU, C. y JANG, S. (2007): Examining E-Relationship Marketing features on hotels websites. Journal of Travel \& Tourism Marketing, nº 21, pp. 33-48. 
BALKA, E. y WAGNER, I. (2006): Making things work: dimensions of configurability as appropriation work. CSCW, pp. 229-238.

BARRANCO, F.J. (2000): Marketing interno y Gestión de Recursos Humanos, Piramide 2000, Madrid.

BECERRIL, D. (2007): TIC y sociedad en el siglo XIX. Universidad de Granada, Facultad de Ciencias, Granada.

BENET, M. (2013): «Facebook para Freelancers (II); 17 técnicas para animar una conversación en tu muro» Socialmediablog.es, el 23 de septiembre del 2014. Disponible en http://socialmediablog.es/facebook-para-freelancers-tecnicas-animar-conversacionmuro-facebook/

BRAUN, P. (2002): "Networking tourism sites: definition, history and scholarship", Journal of Computer-Mediatec Communication, $\mathrm{n}^{\circ} 13$, pp. 210-230.

BULCHAND-GIDUMAL, J.; MELÍAN-GONZALEZ, S. y GONZÁLEZ LÓPEZ-VALCARCEL, B. (2013): “A social media analysis of the contribution of destinations to client satisfaction with hotels", International Journal of Hospitality Management, $\mathrm{n}^{\mathrm{o}}$ 35, pp. 44-47.

CALLARISA, L.; SÁNCHEZ-GARCÍA, J.; CARDIFF, J. y ROSHCHINA, A. (2012): Harnessing social media platforms to measure customer-base hotel brand equity. Tourism Management Perspectives, $\mathrm{n}^{\circ}$ 5, pp. 73-79.

CASTELLS, M. y DIAZ DE ISLA, M.I. (2001): «Diffussion and Uses of Internet in Catalonia and in Spain». IN3 Working Paper, Universitat Oberta de Catalunya. Disponible en http://www.uoc.es/in3/wp/picwp1201.

CHAN, N. y GUILLET, B. D. (2011): «Investigation of social media marketing; how does the hotel industry in Hong Kong perform in marketing on social media websites?» Journal of Travel \& Tourism Marketing, $\mathrm{n}^{\circ}$ 28, pp. 345-368

CLEMENT, A.; CONSTANTINO, T.; KURTZ, D. y TISSENBAUM, M. (2008): Participatory Design and Web 2.0: The Case of PIDWatch, the Collaborative Privacy Toolbar. Proc. PDC, 51-60.

COMISIÓN EUROPEA DE TURISMO (2010): «Online Travel Market» New Media Trend Watch, 21 de octubre del 2014. Disponible en http://www.newmediatrendwatch. com/world-overview/91-online-travel-market?start=2

COX, J. (2009): Using social media to access your customers. Lodging Hospitality, pp. 37.

CUESTA, U. y GASPAR, S. (2013): Aspectos psicosociales, éticos y normativos de la reputación online. Editores Facultad de Ciencias de la Información, Revista Derecom, SSN-e: 1988-2629.

DEL PINO, C. (2007): «Nueva era en la comunicación comercial audiovisual: el marketing viral: Viral marketing and the new age of audiovisual communicatio»Pensar la publicidad: revista internacional de investigaciones publicitarias, Universidad de Valladolid, Valladolid, el 15 de Mayo de 2012. ISSN 1887-8598, Vol. 1, No. 2, Pp. 63-77. Disponible en http://dialnet.unirioja.es/servlet/revista?codigo=11052

DWIVEDI, M., YADAV, A. y VENTKATESH, U. (2012): «Use of social media by national tourism organizations; a prelimiary analysis», Journal of Information Technology \& Tourism, $\mathrm{n}^{\circ} 13$, pp. 93-103.

EHN, P. (2008): Participation in Desing Things. Proc. PDC, pp. 92-101. 
FERRERAS, E.M. (2011): «Redes sociales y cambio social; el movimiento 15M y su evolución en Twitter « Telos; Cuadernos de comunicación e innovación, Fundación Telefónica, Madrid, ISSN 0213-084X, nº 89, pp. 61-73, 12 de Mayo de 2012, Disponible en http://dialnet.unirioja.es/servlet/revista?codigo=6769

FUENTES MORALEDA, L., FIGUEROA DOMEQ, C. Y BALTHASAR, M. (2003): «La distribución electrónica de productos hoteleros: la importancia de los canales basados en Internet», Estudios Turísticos, n ${ }^{\circ}$ 158, pp. 67-78.

FUNDACIÓN BANESTO (2011): Observatorio sobre el uso de las redes españolas en las Pymes españolas. Madrid

GLOBRES (2009): «Do you read your hotel's reviews on TripAdvisor? If not, it's certainly time you did» Globres hotel reservations and marketing Suiza, el 30 de octubre del 2014. Disponible en http://www.globres.com/content.php?page_id=1840

GILBERT, D.; POWELL-PERRY, J. y WIDIJOSO, S. (1999): «Approachs by hotels to the use of the Internet as realtionship marketing tool», Journal of Marketing Practice: Applied Marketing Science, $\mathrm{n}^{\circ}$ 5, pp. 21-38

GONZÁLEZ, F. (2013): «Los hashtags como poderoso recurso en el marketing online; 4 claves»Vuelodigital.com, Buenos Aires, el 25 de febrero del 2013. Disponible en http://www.vuelodigital.com/2013/02/25/los-hashtags-como-poderoso-recurso-en-elmarkting-online-4-claves/

HAGEN, P. y ROBERTSON, P. (2010): Challenges and Opportunities for Participation. T. Social Technologies, proc. PDC, pp. 31-40.

HENDERSON, A. y KYNG, M. (1992): Design at Work. There is No Place Like Home: Continuing Desing in Use. In J. Greembaum y M. Kyng, (Eds.). Lawrence Erlbaum Associates, Hillsdale, New York, pp. 219-240.

HEUNG, V.C.S. (2003): «Barriers to implementing E-commerce in the travel industry: a practical perspective». International Journal of Hospitality Management, $\mathrm{n}^{\circ} 22, \mathrm{pp}$. 111-118.

HOLZNER, S. (2009): Facebook Marketing; Leverage social media to grow your business. Que Publishing, Indianapolis.

HOSTELTUR (2012): «Rentabilidad; asignatura pendiente»VIII Foro Turístico. Hosteltur, Madrid, el 20 de mayo del 2012. Disponible en http://issuu.com/hosteltur_2012/ docs/ebook_viii_foro_hosteltur-rentabilidad_asignatura_/3

HOSTELTUR (2013): «Reportaje; Internet y turismo el campo de batalla cinco años después del boom 2.0» Hosteltur, Madrid, el 28 de enero del 2013. Disponible en http://static.hosteltur.com/web/uploads/2013/01/REPORTAJE_Internet_y_turismo_ el_campo_de_batalla_cinco_anos_despues_del_boom_20.pdf

HSU, Y. L. (2012): «Facebook as international eMarketing strategy of Taiwan hotels», International Journal of Hospitality Management, $\mathrm{n}^{\circ} 31$, pp. 972-980.

JARAMILLO, A.M. (2011): Redes sociales para Todos, Vergara, España.

JOHSON, M. e HYYSALO, S. (2012): Lessons for Participatory Designers of Social Media: Long-Term User Involvement Strategies in Industry. PDC, $\mathrm{n}^{\circ}$ 12, pp. 71-80.

KASAVANA, M.L.; NUSAIR, K. y EODOSIC, K. (2010): «Online social networking: redefining the human web», Journal of Hospitality and Tourism Technology, vol. I, $\mathrm{n}^{\mathrm{o}} 1$, pp. 68-82. 
KOTLER, F. (2004): Los 10 pecados capitales del marketing. Indicios y soluciones Ediciones Gestión 2000. Barcelona

KOTLER, F.; ARMSTRONG, G.; SAUNDES, J. y WONG, V. (2006): Introducción al marketing. Segunda edición europea. Prentice hall. Madrid.

KOTLER, F. y ARMSTRONG, G. (2009): Principios del marketing. Duodécima edición. Person Educación, S. A. Madrid.

KOTLER, F. y KARTAJAYA, H. (2010): Marketing 3.0. Editorial Lid, Madrid.

LI, X. y WANG, Y. (2010): «Evaluating the efectiveness of destination marketing organisation's websites: evidence from China», Intenational Journal of Tourism Research, $\mathrm{n}^{\circ} 12$, pp. 536-549.

MCGUIRRE, K. y NOONE, B. (2014): «How business travelers buy; Hotel pricing in a social world» The Analytic Hospitality Executive, el 17 de octubre de 2014. Disponible en http://blogs.sas.com/content/hospitality/2014/09/02/businesstravelersocialworld/

MARTÍNEZ, S.M, BERNAL, J.J. y MELINAS, J.P. (2012): «Los hoteles de la Región de Murcia ante las redes sociales y la reputación online», Revista de Análisis Turístico, $\mathrm{n}^{\circ} 12$, pp. 1-10.

MELLINAS, J.P., MARTÍNEZ MARÍA DOLORES, S.M. y BERNAL GARCÍA, J.J. (2014): «Uso de las redes sociales en los hoteles de la costa española», Investigaciones Turísticas, $\mathrm{n}^{\circ} 7$, pp. 87-101

MINISTERIO DE INDUSTRIA, COMERCIO Y TURISMO (2015): Balance del año turístico 2014. Publicado el 22 de enero de 2015. Disponible en http://www.minetur.gob.es/es-es/gabineteprensa/notasprensa/2015/documents/150121dossier\%20 balance\%20tur\%C3\%ADstico\%202014.pdf

MÖRCH, A. I. (1997): Method and tool for tailoring of object oriented applications: an evolving artifacts approach. Unpublished doctoral dissertation. University of Oslo, Norway.

MUÑOZ, A. y ARGÜELLES, I. (2010): «Análisis del discurso en redes sociales. Twitter un caso bajo estudio» Analizar datos, Universidad de Vigo, pp. 64, Vigo, el 13 de mayo del 2012. Disponible en http://dialnet.unirioja.es/servlet/libro?codigo=433629.

NARDI, B., (1993): A Small matter of programming: perspectives on end user computing. Mit Press, Cambridge, MA, USA.

PÉREZ, M., MARTÍNEZ, A. y ABELLA, S. (2002): «El sector Turístico y el Comercio electrónico», Boletín Económico de ICE, n 2733, pp. 31-37.

VIRGINIA PHELAN, K., CHEN, H. T., y HANEY, M. (2013): «"Like” and "Check-in": how hotels utilize Facebook as an effective marketing tool» Journal of hospitality and Tourism Technology, $\mathrm{n}^{\circ}$ 4(2), pp. 134-154.

ROSERO, S. (2013): 14 cuentas españolas de transportes, viajes y turismo en twitter a examen. Centro Tadel Formacio y MKtFan, Barcelona.

SENA, K.; MCNELLIS, A. y MCDADE, C. (2014): «Google's Travel Plan in a post-atomic era» Evercore, el 17 de octubre del 2014. Disponible en http://www.evercore.com SIVERA, S. (2008): Marketing viral, UOC, Barcelona.

STAMBOULIS, Y. y SKAYANNIS, P. (2003): «Innovation strategies and technology for Experience-based tourism», Tourism Management, $\mathrm{n}^{\circ}$ 24, pp. 35-43. 
SULÉ, M.A. Y PRIETO, J. (2010): «MK-2.0 secretos a voces del social-media» Revista de la Facultad de Ciencias Económicas y Empresariales, Pecunia, ISBN: 699-9495, $\mathrm{n}^{\circ}$ Extra 1, pp. 191-214. León, el 15 de junio del 2013. Disponible en http://dialnet. unirioja.es/servlet/articulo?codigo $=3315326$

TREADAWAY, C. y SMITH, M. (2010): Facebook Marketing; An hour a day. Wiley Publishing, Inc., Indianápolis, IN.

TWITTER (2014): El 26 de octubre del 2014. Disponible en http://www.twitter.com

VERHOEF, P.C.; REINARTZ, W.J. y KRAFFT, M. (2010): «Customer engagement as a new perspective in customer management», Journal of Service Research, vol. 13, $\mathrm{n}^{\mathrm{o}}$ 3, pp. 247-251.

VILLALBA TRUJILLO, R.; MARTÍNEZ CARO, L. MARTÍNEZ MARÍA-DOLORES, S.M. (2014): «Análisis regional de las empresas turísticas on line en España», Cuadernos de Turismo, $\mathrm{n}^{\mathrm{o}} 34$, pp. 335-349.

VOSS, A. (2009): Configuring User-Designer Relations: Interdisciplinary Perspectives. Springer, London.

XIANG, Z. y GREZTEL, U. (2010): «Role of social media in online travel information search», Tourism Management, $\mathrm{n}^{\circ} 31$, pp. 179-188.

YE, Q.; LAW, R.; GU, B. y CHEN, W. (2011): «The influence of suer-generated content on traveler behaviour: an empirical investigation on the effects of e-wor-of-mouth to hotel online bookings », Computers in Human Behavior, $\mathrm{n}^{\circ}$ 27, pp. 634-639.

ZARRELLA, D. (2010): The Social Media Marketing Book. O’Reilly Media, Inc. Sebastapol, C.A. 